\title{
Dyslipidemia: another brick in the wall. A feasible link in the OSA-cardiovascular disease axis
}

\author{
János Juhász
}

Received: 17 May 2012 /Revised: 11 April 2013 / Accepted: 17 April 2013 / Published online: 16 May 2013

(C) Springer-Verlag Berlin Heidelberg 2013

Obstructive sleep apnea (OSA) is a frequent and often severe clinical condition that has been linked to cardiovascular diseases and metabolic disorders among others. The underlying mechanisms initiating and aggravating cardiovascular disorders are not clearly understood yet, oxidative stress and endothelial dysfunction are considered as major factors. A frequent consequence of OSA is diminished endothelial function including decreased nitric oxide (NO)-dependent vasodilation. A very recent review summarizing current experimental information from animal models gives a deep insight in the complexity of alterations in $\mathrm{NO}$ production in patients with OSA [1].

Dyslipidemia (high serum concentrations of total cholesterol, low-density lipoprotein cholesterol (LDL-C), highdensity lipoprotein cholesterol (HDL-C), and triglycerides (TG)) is considered as one of the most important risk factors for cardiovascular disease, most notably for coronary artery disease (CAD) and is target for therapeutic intervention.

The investigations of pathomechanisms both for OSA and dyslipidemia impacting cardiovascular risk are now accomplished on the biochemical/molecular level. Consequently, the link between OSA and dyslipidemia also has been (and to be) investigated on the biomolecular level. If we consider the whole complexity of mechanisms, we may not disregard the underlying/possible genetic influences that may further complicate the interactions.

Since the genetic code has been cracked, a lot of efforts have been made to find the underlying genetic basis for common diseases. Common diseases are polygenic and now we know that each contributing gene has a very small

\section{J. Juhász $(\square)$}

Pneumologie, Beatmungs- und Schlafmedizin,

Zentrum Innere Medizin, Klinikum Werra-Meißner GmbH,

Elsa-Brändström Str. 1,

37269 Eschwege, Germany

e-mail: juhasz_j@yahoo.de effect which cannot be mapped by linkage in families. Over a thousand loci are now associated with some 150 common diseases and traits. Most diseases are influenced by many loci, but the majority of the variants have modest effects, increasing the risk by $10-50 \%$ [2]. Using a genome-wide association study for serum lipids in $>100,000$ individuals Teslovich and colleagues identified 95 loci that showed significant association $\left(P<5 \times 10^{-8}\right)$ with at least one or more of the four lipid or lipoprotein classes tested (total cholesterol, low-density lipoprotein cholesterol, high-density lipoprotein cholesterol, triglycerides) [3]. Some of these loci are associated not only with lipids but also with risk for CAD. This suggests that there may be selective mechanisms by which HDL-C or TG can be altered in ways that also modulate CAD risk.

However, it is also possible that causal genes in these loci may have pleiotropic effects on non-lipid parameters that are causal for CAD risk reduction. For example, the major allele of the lead single-nucleotide polymorphisms (SNP) in the IRSI locus is associated with increased risk of type 2 diabetes mellitus, insulin resistance, and hyperinsulinemia [4], along with decreased HDL-C, increased TG, and increased risk of $\mathrm{CAD}$. It remains unclear which of the metabolic risk factors are responsible for the increased CAD risk.

In the only paper on the issue, Nayazit et al. reported that the polymorphism of the IRS-1 gene at codon 972, especially Gly/Arg variant, or the presence of the allele for Arg appears to be associated with occurrence of OSA in male patients, whereas this polymorphism is not related to severity of OSA [5]. Interestingly, there was no relationship between the genotypes and diabetes mellitus. The authors compared the founding of 50 patients and 143 healthy controls.

Now, it is widely accepted that metabolic syndrome (comprising dyslipidemia) promotes atherosclerosis, the major contributing factor for coronary heart disease. 


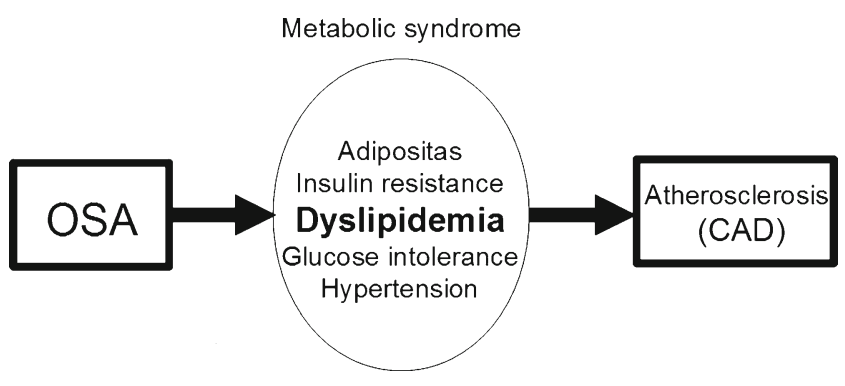

Fig. 1 The OSA-cardiovascular disease axis. Bold arrow indicates promoting effect. For abbreviations please refer to the text

Increasing evidence from animal models of OSA shows that intermittent hypoxemia is independently associated with metabolic syndrome, particularly with regard to increase in serum cholesterol and phospholipids, upregulation of pathways of triglyceride and phospholipid biosynthesis and inhibition of mechanisms for cholesterol uptake by the liver [6]. It is a reasonable and compelling consideration that the feasible chain link from OSA to cardiovascular disease (CAD) leads along the pathways through metabolic syndrome (dyslipidemia) (Fig. 1).

In this issue of Sleep and Breathing, Adedayo and colleagues present a review paper describing the possible links between OSA and dyslipidemia [7]. Several review and original papers has been published to elucidate the underlying mechanism between OSA and metabolic syndrome/dyslipidemia (PubMed indicates 208 references!).

What is new in this article compared to previous review papers and what adds the paper to our present knowledge? The authors line up all the possible mechanisms that could account for the underlying mechanisms. They extensively discuss all the effects of hypoxia on different enzymes and show that chronic intermittent hypoxia has a substantial impact on lipid metabolism resulting in hyperlipidemia/dyslipidemia. Also, the authors provide a schematic figure to summarize the main steps of the complex interactions and make the mechanisms more understandable. Adedayo and colleagues put the tesserae of a realistic picture together by trying to establish a plausible link between OSA and dyslipidemia based on recent experimental animal model.

An advantage that further strengthens the paper is without doubt the additional information on the underlying mechanisms in cases without intermittent hypoxemic events and the indication, how these apply to the relationship between OSA and dyslipidemia.
In their conclusion, the authors resist the apparent temptation, namely to present the enumerated mechanisms as established link. They leave the question-quite so-open.

Do we get in the end the proof of evidence? Certainly not. But the demonstration of the possible underlying mechanisms is very compelling and conclusive. Another brick in the wall.

Who should read this article? All of you, who look for an intellectual challenge, who like to put the pieces of a puzzle together without having a master picture to follow. It is not an easy-reading evening lecture. But in the end, you will have a proper insight in the complexity of the interactions between sleep-related breathing disorders, metabolic conditions (dyslipidemia), and the number one killer-coronary heart disease.

Conflict of interest The author declares that he has no conflict of interest in relationship with this article.

\section{References}

1. Golbidi S, Badran M, Ayas N, Laher I (2012) Cardiovascular consequences of sleep apnea. Lung 190:113-132

2. Scott J, Thompson G (2012) The discovery of the structure of DNA. In: Thompson G (ed) Nobel prizes that changed medicine. Imperial College Press, London, pp 89-111

3. Teslovich TM, Musunuru K, Smith AV et al (2010) Biological, clinical and population relevance of 95 loci for blood lipids. Nature 466:707-713

4. Rung J, Cauchi S, Albrechtsen A, Shen L, Rocheleau G, CavalcantiProença C, Bacot F, Balkau B, Belisle A, Borch-Johnsen K, Charpentier G, Dina C, Durand E, Elliott P, Hadjadj S, Järvelin MR, Laitinen J, Lauritzen T, Marre M, Mazur A, Meyre D, Montpetit A, Pisinger C, Posner B, Poulsen P, Pouta A, Prentki M, Ribel-Madsen R, Ruokonen A, Sandbaek A, Serre D, Tichet J, Vaxillaire M, Wojtaszewski JF, Vaag A, Hansen T, Polychronakos C, Pedersen O, Froguel P, Sladek R (2009) Genetic variant near IRS1 is associated with type 2 diabetes, insulin resistance and hyperinsulinemia. Nat Genet 41:1110-1115

5. Bayazit YA, Erdal ME, Yilmaz M, Ciftci TU, Soylemez F, Gokdogan T, Kokturk O, Kemaloglu YK, Koybasioglu A (2006) Insulin receptor substrate gene polymorphism is associated with obstructive sleep apnea syndrome in men. Laryngoscope 116(11):1962-1965

6. Li J, Thorne LN, Punjabi NM, Sun CK, Schwartz AR, Smith PL, Marino RL, Rodriguez A, Hubbard WC, O'Donnell CP, Polotsky VY (2005) Intermittent hypoxia induces hyperlipidemia in lean mice. $\mathrm{v}$ 97:697-706

7. Adedayo M, Olafiranye O, Smith D, Hill A, Zizi F, Brown C, JeanLouis D (2012) Obstructive sleep apnea and dyslipidemia: evidence and underlying mechanisms. Sleep Breath. doi:10.1007/s11325012-0760-9 